\title{
Why some parents made firearms more accessible during the beginning of the COVID-19 pandemic: results from a national study
}

\author{
Rebeccah L. Sokol ${ }^{1}$ • Lea Marineau ${ }^{2} \cdot$ Marc A. Zimmerman $^{3,4,5} \cdot$ Laney A. Rupp ${ }^{3,5}$. \\ Rebecca M. Cunningham ${ }^{3,4,6} \cdot$ Patrick M. Carter ${ }^{3,4,6}$
}

Received: 19 January 2021 / Accepted: 6 July 2021 / Published online: 23 July 2021

(C) The Author(s), under exclusive licence to Springer Science+Business Media, LLC, part of Springer Nature 2021

\begin{abstract}
The objective of this study was to assess parents' firearm storage behaviors during the COVID-19 pandemic and characterize reasons why some parents made their firearms more accessible during this time. In June-July 2020, the study team conducted the FACTS National Survey-a cross-sectional, web-based, survey of 2,924 parents and their teens (ages14-18) regarding firearm-related practices. We weighted descriptive analyses to be nationally representative of parents of teens in the United States. We utilized qualitative thematic analysis to identify parents' reasons for making firearms more accessible. Five percent of firearmowning parents of teens reported making their firearms more accessible during the beginning of the COVID-19 pandemic. Reasons why parents increased the ease of firearm access included: (1) Increased civil unrest and riots; (2) Threat of home invasion and/or crime victimization; (3) Fear of panic
\end{abstract}

Rebeccah L. Sokol

rlsokol@wayne.edu

1 School of Social Work, Wayne State University, 5447 Woodward Ave, Detroit, MI 48202, USA

2 School of Nursing, Johns Hopkins University, 525 N Wolfe St, Baltimore, MD 21205, USA

3 Department of Health Behavior and Health Education, School of Public Health, University of Michigan, 1415 Washington Heights, Ann Arbor, MI 48109-2029, USA

4 University of Michigan Injury Prevention Center, 2800 Plymouth Road, NCRC 10-G080, Ann Arbor, MI 48109, USA

5 Michigan Youth Violence Prevention Center, School of Public Health, University of Michigan, 1415 Washington Heights, Ann Arbor, MI 48109-2029, USA

6 Department of Emergency Medicine, University of Michigan School of Medicine, 1500 East Medical Center Drive, Ann Arbor, MI 48109, USA and the unknown; and (4) Easier access and greater protection, threat unspecified. Some parents-largely motivated by fear-chose to store firearms in a more accessible manner during the beginning of the COVID-19 pandemic to protect their family against possible external threats. Understanding the fear that motivates parents' decisions regarding storage practices might aid interventions focused on harm reduction and safer storage.

Keywords Firearms · COVID-19 · Parents · Injury prevention $\cdot$ Violence prevention $\cdot$ Firearm safety

\section{Introduction}

Following the onset of the coronavirus disease 2019 (COVID-19) pandemic, Americans experienced multiple causes of unrest, including community spread of the virus and its associated morbidity and mortality (Bartsch et al., 2020; Weinberger et al., 2020), high levels of unemployment (Coibion et al., 2020), the exacerbation of social inequities (Gray et al., 2020; Pirtle, 2020; van Dorn et al., 2020), declining mental health (Czeisler et al., 2020; Galea et al., 2020; Holmes et al., 2020), and increased attention to the over-policing of and police violence against Black Americans (Bresler \& Beletsky, 2020). Taken together, the COVID-19 era is defined by tumult that extends beyond the virus. Firearm sales rise when Americans experience heightened real and perceived threats and corresponding desires for protection (Lang \& Lang, 2020). Likewise, firearm sales in the United States steeply rose during the beginning of the COVID-19 pandemic - more so than other events, including the September 11th terrorist attacks and the Sandy Hook Elementary school shooting (Brauer n.d.; Lang \& Lang, 2020). 
With a greater number of firearms in homes, it is necessary to identify how firearm owners-especially parentsstore firearms in an effort to prevent injury and death. In general, individuals who keep firearms for protection are less likely to store firearms unloaded and locked compared to individuals who own firearms for recreational purposes, such as hunting or sport-shooting (Vacha \& McLaughlin, 2000; Wiktor et al., 1994). Safe storage is a key strategy to prevent firearm-related injuries among youth populations. Risk of intentional and unintentional firearm-related injury and death in youth are associated with storage practices, and risk increases when firearms are easily accessible due to storing firearms in an unlocked and/or loaded manner (Monuteaux et al., 2019). Thus, changes in firearm storage that make firearms more readily accessible may have important implications for youth health and safety.

Firearm-related injuries are the second leading cause of death among children and adolescents (1-19 years old) (Cunningham et al., 2018) and the leading cause of death among emerging and young adults (15-24 years old) (Kochanek et al., 2019). The convergence of the COVID19 pandemic, racial unrest, and increased threat and distress might have exacerbated firearm-related injuries and deaths among youth on a few different fronts. First, many youth spent more time at home given school closures at the beginning of the pandemic, and some may have been unsupervised while their parents worked (either remotely or inperson) (Armitage \& Nellums, 2020). This unsupervised time may have increased the risk of unintentional firearm injury. Second, adolescent and young adult depression, anxiety, suicide ideation, and suicide attempts increased following the start of the COVID-19 pandemic (Hawes et al., 2021; Hill et al., 2021), raising concerns about self-inflicted injury in settings where a firearm is easily accessible. Third, the convergence of increased stress and more time at home led to a rise in intimate partner violence (Boserup et al., 2020); the presence of an accessible firearm in a setting with intimate partner violence can increase the potential for a lower level fight to escalate into a lethal event, exposing children to witnessing fatal violence or experiencing bystander violent victimization. These combined forces highlight that parents' firearm storage behaviors may have serious health and safety implications for youth living at home.

To inform firearm injury prevention during times of crisis, it is critical to understand if parents made changes to their firearm storage practices during the COVID19 pandemic, and if so, the underlying motivations for such changes. The present research addresses this gap by assessing parents' firearm storage behaviors during the COVID-19 pandemic and probing reasons why some parents elected to make their firearms more accessible during this time.

\section{Methods}

\section{Data}

In June and July 2020, the study team conducted the FACTS National Survey, which was a cross-sectional, webbased, survey of 2,924 U.S. parents and their teens (ages 14-18 years) regarding firearm-related practices (FACTS National Survey User Guide, 2020). We defined parents as the primary caretakers of teens (e.g., parent, step-parent, grandparent; hereafter referred to as 'parent'). Participants for the study were from the Gallup Panel, a probability-based panel that is constructed to be broadly representative of the U.S. adult population. Panelists are recruited using random digit-dial phone interviews that cover landline and cell phones and address-based sampling methods. Panel weights assigned to each respondent incorporated unequal selection probabilities and made analyses nationally representative of parents of teens in the United States with respect to parent age, gender, education, race, ethnicity, census region, as well as child age and gender. We utilized these weights in all quantitative analyses. Respondents received $\$ 5$ for completing the online survey. The University of Michigan Institutional Review Board approved this study. The present analyses use parent responses to identify which parents made changes to firearm storage practices following the start of the COVID-19 pandemic and why.

\section{Measures}

Household firearm ownership To assess if a household member owned a firearm, parents answered: "Do you personally own a gun?" and "Does anyone else you live with own a gun?" Answering 'yes' to either question indicated household firearm ownership, hereafter referred to as 'firearmowning parent.'

Firearm storage We asked the sub-set of firearm-owning parents $(n=1,452)$ from the larger parent sample $(\mathrm{N}=2,924)$ about their firearm storage behaviors. Specifically, we asked parents if any of the firearms on their property are stored loaded (e.g. with the ammunition in the magazine, revolver cylinder, and/or chamber of the gun), if any of the firearms on their property are stored unlocked (e.g., don't need to have a key or combination to get to the gun[s]), and if any of the firearms on their property are stored both unlocked AND loaded.

Firearm storage changes We also asked firearm-owning parents: "Do you store any guns on your property differently than you did before the COVID-19 pandemic?" Among parents who indicated they stored firearms differently, we asked: "Compared to before the COVID-19 pandemic, are any of these guns stored in a way that makes them more accessible?" (Response: Yes/No); and "Compared to before 
the COVID-19 pandemic, are any of these guns stored in a way that makes them less accessible?" (Response: Yes/No). Among parents who indicated that they stored their firearms differently since the start of the pandemic, we asked: "What is the most important reason for changing your gun storage practices since the start of the COVID-19 pandemic?" Parents answered in free-text form. Because only eight parents reported they made their firearms less accessible, we presently focus on parents who reported making their firearms more accessible.

Other firearm-related behaviors To assess firearm type(s) present in the home, we asked firearm-owning parents what type of gun(s) they owned and what type of gun(s) other members of their household owned. Non-mutually exclusive response options included handgun and long gun. We also asked firearm-owning parents if, since the beginning of the COVID-19 pandemic they: (1) purchased a firearm; and (2) purchased more ammunition than typical.

Socio-demographic variables We assessed parents' sociodemographic characteristics. Specifically, we collected information on parent biological sex, race, age, education, and political affiliation. We also collected information regarding perceptions about community violence exposure using items from the "Things I have seen and heard" survey (Richters \& Saltzman, 1990). Specifically, we asked parents if, in the past 12 months, they had seen or heard any of the following in their neighborhood: (1) guns being shot; (2) somebody arrested; (3) drug deals; (4) somebody get beaten up; (5) somebody be stabbed; (6) somebody pull a knife on another person; (7) somebody pull a gun on another person; (8) somebody get shot; (9) my house was been broken into. We classified parents who endorsed any of the items as having witnessed community violence.

\section{Analysis}

We conducted Pearson's chi-square tests to identify if firearm-related behaviors, witnessing community violence, and other socio-demographic characteristics differed among firearm-owning parents who did versus did not make their firearms more accessible after the onset of the pandemic. To explore motivations underlying changes in firearm storage behaviors, our qualitative sample included firearm-owning parents who indicated they stored their firearms in a more accessible way since the start of the pandemic and provided a reason $(n=61)$. Two authors utilized inductive coding to independently generate themes regarding why parents stored their firearms in a more accessible manner since the start of the COVID-19 pandemic. The authors met to review their independently generated themes and develop consensus on final themes. A third author reviewed themes to ensure they fully covered all topics that emerged from parent responses.
Two authors then independently assigned each parent response to one or more thematic codes and resolved any discrepancies through discussion until reaching consensus.

\section{Results}

Seventy parents, $5 \%$ of all firearm-owning parents, reported that they made changes to their firearm storage practices since the start of the COVID-19 pandemic that increased ease of firearm access. Table 1 provides characteristics of the sub-sample of firearm-owning parents who made their firearms more accessible $(n=70)$ compared to firearm-owning parents who did not make their firearms more accessible since the pandemic $(n=1,382)$. Parents who made their firearms more accessible were younger, on average, compared to parents who did not (42 versus 47 years old, respectively, $p=0.02$ ). Parents who made their firearms more accessible since the start of the pandemic also were more likely than parents who did not make them more accessible to have purchased a firearm $(\mathrm{X} 2[1, \mathrm{~N}=1452]=4.0, p=0.047)$ and more ammunition than typical $(\mathrm{X} 2[1, \mathrm{~N}=1452]=9.1, p=0.003)$ since the start of the pandemic, and reported having exposure to community violence in the past 12 months (X2[1, $\mathrm{N}=1452]=6.0, p=0.01$ ).

Among the parents who reported that they made changes to storage practices since the start of the COVID-19 pandemic to make their firearms more accessible, 61 parents provided an answer to: "What is the most important reason for changing your gun storage practices since the start of the COVID-19 pandemic?" Four distinct themes emerged from our inductive thematic coding process of parent responses to this question. Motivations for increasing accessibility since the start of the COVID-19 pandemic included: (1) Increased civil unrest and riots; (2) Threat of home invasion and/or crime victimization; (3) Fear of panic and the unknown; and (4) Easier access and greater protection, threat unspecified.

Increased civil unrest and riots. Almost half of participants $(n=29)$ cited concerns about civil unrest or riots as the most important reason for changing their firearm storage practices since the start of the COVID-19 pandemic. A nonspecific, generalized fear of riots and civil unrest comprised the majority of these responses. Some respondents did not include details as to the origin of this unrest and answered with short statements such as "civil unrest," "concerns with unrest," "mob mentality," or "riots."

In contrast to the majority of respondents who did not elaborate on the origins of the unrest, five participants made specific references to race-based violence or the Black Lives Matter movement. One respondent wrote that they changed their firearm storage practice because of fears of white supremacists. The current divided political climate and influence of political officials was described as a concern 
Table 1 Descriptive statistics for firearm-owning parents who (a) did not and (b) did make their firearms more accessible since the beginning of the COVID-19 pandemic

\begin{tabular}{|c|c|c|c|}
\hline & $\begin{array}{l}\text { Firearm-owning parents who did not make } \\
\text { their firearms more accessible since the } \\
\text { pandemic } \\
(\mathrm{n}=1382)\end{array}$ & $\begin{array}{l}\text { Firearm-owning parents who made their } \\
\text { firearms more accessible since the pan- } \\
\text { demic } \\
(\mathrm{n}=70)\end{array}$ & $p$ \\
\hline Store $\geq 1$ firearm loaded OR unlocked & $69 \%$ & $77 \%$ & 0.45 \\
\hline Store $\geq 1$ firearm loaded AND unlocked & $26 \%$ & $36 \%$ & 0.37 \\
\hline $\begin{array}{l}\text { Purchased a firearm since the beginning of } \\
\text { the pandemic }\end{array}$ & $17 \%$ & $39 \%$ & 0.047 \\
\hline $\begin{array}{l}\text { Purchased more ammunition than usual since } \\
\text { the beginning of the pandemic }\end{array}$ & $18 \%$ & $61 \%$ & 0.003 \\
\hline Handgun ownership & $81 \%$ & $88 \%$ & 0.28 \\
\hline Long gun ownership & $78 \%$ & $76 \%$ & 0.80 \\
\hline Community violence exposure & $50 \%$ & $76 \%$ & 0.01 \\
\hline Age $(\bar{x}[S E])$ & $47.2(0.5)$ & $42.1(2.1)$ & 0.02 \\
\hline Sex & & & 0.90 \\
\hline Male & $52 \%$ & $53 \%$ & \\
\hline Female & $48 \%$ & $47 \%$ & \\
\hline Race & & & 0.92 \\
\hline Non-Hispanic White & $78 \%$ & $70 \%$ & \\
\hline Non-Hispanic Black or African American & $10 \%$ & $9 \%$ & \\
\hline Hispanic & $11 \%$ & $21 \%$ & \\
\hline Other & $<0.01 \%$ & - & \\
\hline Education & & & 0.25 \\
\hline $\begin{array}{l}\text { Less than high school or high school gradu- } \\
\text { ate }\end{array}$ & $34 \%$ & $24 \%$ & \\
\hline Some college, trade school, or 2-year degree & $33 \%$ & $41 \%$ & \\
\hline College graduate: 4-year degree & $15 \%$ & $22 \%$ & \\
\hline Postgraduate or professional schooling & $18 \%$ & $13 \%$ & \\
\hline Political affiliation & & & 0.12 \\
\hline Democrat & $26 \%$ & $9 \%$ & \\
\hline Republican & $46 \%$ & $58 \%$ & \\
\hline Independent & $25 \%$ & $27 \%$ & \\
\hline Other & $5 \%$ & $8 \%$ & \\
\hline
\end{tabular}

Estimates weighted to represent national statistics. $p$-values based on Pearson's chi-square test with the Rao-Scott adjustment (for categorical variables) and t-tests (for continuous variables) comparing parents who did not and did make changes to their firearm storage. Bold indicates significant differences at $\alpha=0.05$

for inciting violence towards under-represented racial and ethnic groups.

As a person of color when I hear the US President vilifying immigrants and people of color and then when I see White supremacists taking over public buildings and streets I genuinely fear for my children's life and my own. I refuse to go down without a fight.

In contrast, four participants described threat or fear from Black Lives Matter protestors or Black people as their most important reason for changing their firearm storage. Two responses included racist language that were explicit in their references to targeting Black people. These responses highlighted the racialization of blame for riots or protests on under-represented racial and ethnic groups, specifically Black Americans. These responses also echoed conspiracy theories aimed at fueling fears about racial conflict.

Make them [firearms] more accessible if defense against Black mobs becomes necessary.

Riots and threats of Black-on-white ethnic cleansing.

The remaining two responses describing threat or fear from Black Lives Matter protestors or Black people lacked description; however, unlike the responses that only stated riots or civil unrest as their most important reason, these responses went further by specifically stating the Black Lives Matter movement: "Rioting near us during Black Lives Matter protests." Although no further description 
was provided, the mention of Black Lives Matter may demonstrate a perceived threat of social justice movements centered on under-represented racial and ethnic groups and the fear of resulting violent disturbances. Thirteen (44.8\%) responses contained the term "riot", while three (10\%) contained the term "protest."

Threat of home invasion and/or crime victimization. Fourteen respondents (23\%) reported that an increased threat of home invasion and crime victimization during the beginning of the COVID-19 pandemic was the main reason why they stored their firearms in a more accessible manner. Many respondents alluded to high levels of unemployment and shortages in food and supplies as a result of the COVID-19 pandemic serving as the impetus for home invasion:

In general, supplies were becoming less accessible to the general public making theft more likely.

Other respondents alluded to fearing victimization, but they did not specify the COVID-19 pandemic as the source of increased crime:

More thieves with nothing else to do but steal from me and my neighbors.

Overall, these respondents feared personally-targeted crimes-such as home invasion and burglary-which differs from general community-based violence.

Fear of panic and the unknown. In addition to civil unrest-specific and home-based crime reasons for making firearms more accessible, many parents $(n=14 ; 23 \%)$ cited general fear as a reason for increasing firearm accessibility, including fear of the unknown, fear of strangers, and fear of mass panic. One parent answered:

\section{$[\ldots]$ fear of the world.}

Overall, these parents were largely characterized by a general fear of uncertainty-including political uncertainty and pandemic-related uncertainty - and not knowing how others will react to such uncertainty.

Easier access and greater protection, threat unspecified. Every participant in this analysis has changed the way they store their firearms since the COVID-19 pandemic; however, 11 (18\%) participants stated ease of access and/or greater protection as their primary reason for this change. Although they may have had other motivations, access and/or protection was the only response they indicated. This theme was mutually exclusive, and participants did not specify the exact threat. Statements included: "quick access," "extra protection," "easier access," and "to make them more accessible in an emergency."

\section{Discussion}

Overall, parents who reported that they changed their firearm storage to make their firearms more accessible cited fear or a safety concern. Thus, fear and safety concerns appear to promote ease-of-access firearm storage among a nontrivial portion of firearm-owning families. Estimates suggest that around 20 million youth in the United States live in a household with a firearm (Azrael et al., 2018). Extrapolating our present results to this larger population would imply that over one million youth in the United States are living in households where parents are storing firearms in a more accessible manner since the start of the COVID-19 pandemic.

Paradoxically, some parents' perceptions that the outside world is threatening their families' safety is motivating changes in firearm storage practices within the home that increases a known risk to their teens' safety. Not only were some parents making firearms more accessible within their homes, but we found that these parents were also more likely to engage in other firearm-related behavior changes-including purchasing one or more firearms and more ammunition than typical-since the start of the pandemic. Our present findings expand our understanding of how storage practices change alongside elevated purchasing rates during uncertain times. Specifically, we identified a pattern of behavior in which increased firearm and ammunition purchasing are occurring in tandem with unsafe firearm storage practices within families. Given that firearm availability is a known risk factor for unintentional injuries among younger children and a risk factor for adolescent suicide, this purchase-storage pattern may elevate risk for youth firearm-related injury and death (Anglemyer et al., 2014; Dahlberg et al., 2004; Grossman et al., 2005; Mathew Miller et al., 2002; Matthew Miller et al., 2005; Monuteaux et al., 2019).

Firearm-owning parents may be conducting their own risk-benefits analyses, and some parents ultimately favor easy firearm access for protection over locked and unloaded firearm storage. Researchers have reported that these parents might not be fully considering the risks of easily accessible firearms (Conner et al., 2018). A large body of evidence has demonstrated the presence of a firearm in a teen's home substantially increases the risk of suicide and unintentional firearm death, and this risk increases in homes where the firearm is stored in a more accessible manner (e.g., loaded and unlocked) (Anglemyer et al., 2014; Branas et al., 2009; Dahlberg et al., 2004; Grossman et al., 2005; Mathew Miller et al., 2002; Matthew Miller et al., 2005; Monuteaux et al., 2019). Recent research also suggests that firearm owners have mixed appreciation of this risk (Conner et al., 2018). Moreover, public opinion regarding the risks and benefits of having a firearm in the home has evolved over time. In 2000, approximately $35 \%$ of US adults believed that a gun in the 
home makes it a safer environment, whereas by $2014,63 \%$ did (McCarthy, 2014).

Parents who have elected to store their firearms in a more accessible manner largely do so to protect their family against potential threats outside the home. Intervention strategies focused on increasing safe firearm storage practices should leverage this common goal of safety and the importance of family. Yet, public health messaging of safety and threat can be at odds with parents' ideas of safety and threat. Traditional messaging about firearm storage and safety has focused on the firearm as a threat to child health and safety, whereas parents may view the firearm as a protector of their child's health and safety. To achieve overall safer environments for youth, more nuanced public health messaging may be needed that goes beyond educating parents to store their firearms unloaded and locked and addresses the underlying motivations for parents' safety concerns and fears and how safer storage aids in protecting their family.

The plurality of respondents cited civil unrest as the main source of fear motivating them to make firearms more accessible, and a few respondents used overt racist language. Greater attention needs to be given to individual and community-wide interventions addressing underlying issues of racism. In addition to perceived threats motivating less safe firearm storage, we found that parents who were more likely to change firearm storage to be more accessible also reported more perceived community violence exposure. Thus, our results suggest that youth firearm violence and injury prevention may benefit by encompassing a comprehensive approach that both helps address perceived threats and focuses attention on community violence. Although these findings may be particular to this moment in time, it is nevertheless informative to understand how current events such as the recent pandemic and social unrest may be motivating factors for changing storage practices to unsafe conditions.

As a nationally representative study conducted during a unique time of the COVID-19 pandemic, national unrest and demonstrations in multiple cities, and the run-up to a national election, this is the first research to deeply investigate firearm storage behaviors during a period of increased firearm ownership and purchasing. Our estimate that around half of parents of teenagers own a firearm is higher than previous national studies that report around one-third of parents keep a gun in or around their homes (Azrael et al., 2018; Hamilton et al., 2018). We theorize this discrepancy is partly due to sample definition. Presently, we focus on parents of teens, whereas previous estimates focus on parents of all children. Thus, firearm ownership may be more prevalent among parents of teens compared to parents of younger children. Additionally, estimates suggest 2.1 million excess firearms were purchased from March through May 2020—a 64.3\% increase over expected volume (Schleimer et al., 2020). This increase in sales since the beginning of the COVID-19 pandemic may also contribute to the difference in estimates from prior studies. Because of the intertwined issues present during the investigated time period, findings may not generalize to other times of uncertainty or unrest. Finally, the majority of firearm-owning parents did not change their storage practices since the onset of the COVID-19 pandemic. Our findings suggest that future studies that investigate why parents maintain the same firearm storage practiceswhether stored unlocked or locked, loaded or unloaded-in the presence of external disruptions would be useful.

\section{Conclusion}

Our combination of quantitative and qualitative inquiry provides us with inference as to why parents changed firearm storage behaviors during the beginning of the COVID-19 pandemic. Some parents-largely motivated by fear-chose to store firearms in a more accessible manner to protect against possible external threats. Encouraging parents to reassess the risks that accessible firearm storage pose to their teens might help parents favor locked and unloaded firearm storage.

Author contributions Dr. Sokol was involved in survey design, and Dr. Sokol developed and led analysis and manuscript writing. Drs. Zimmerman, Carter, and Cunningham led survey design and data collection and contributed to the writing and editing of the manuscript. Ms. Rupp and Ms. Marineau were involved in survey design and data collection, and they contributed to qualitative coding, manuscript writing, and editing.

Funding Data collection for this project was funded through the National Institutes of Health, National Institute of Child Health and Human Development (1R24HD087149-01A1).

\section{Declarations}

Conflict of interest The authors have no conflicts of interest to disclose.

Consent to participate Participants provided informed consent prior to completing the questionnaire.

Consent for publication Participants provided informed consent prior to completing the questionnaire.

Ethics approval The University of Michigan Institutional Review Board approved this study.

Human and Animal Rights and Informed Consent The University of Michigan IRB approved the study and participants provided informed consent prior to completing the questionnaire. 


\section{References}

Anglemyer, A., Horvath, T., \& Rutherford, G. (2014). The Accessibility of firearms and risk for suicide and homicide victimization among household members: A systematic review and meta-analysis. Annals of Internal Medicine, 160, 101-110. https://doi.org/ 10.7326/M13-1301

Armitage, R., \& Nellums, L. B. (2020). Considering inequalities in the school closure response to COVID-19. The Lancet Global Health, 8, e644.

Azrael, D., Cohen, J., Salhi, C., \& Miller, M. (2018). Firearm storage in gun-owning households with children: Results of a 2015 national survey. Journal of Urban Health, 95, 295-304.

Bartsch, S. M., Ferguson, M. C., McKinnell, J. A., O'Shea, K. J., Wedlock, P. T., Siegmund, S. S., \& Lee, B. Y. (2020). the potential health care costs and resource use associated with COVID-19 in the United States: A simulation estimate of the direct medical costs and health care resource use associated with COVID-19 infections in the United States. Health Affairs, 39, 927-935.

Boserup, B., McKenney, M., \& Elkbuli, A. (2020). Alarming trends in US domestic violence during the COVID-19 pandemic. The American Journal of Emergency Medicine, 38, 2753-2755.

Branas, C. C., Richmond, T. S., Culhane, D. P., Ten Have, T. R., \& Wiebe, D. J. (2009). Investigating the link between gun possession and gun assault. American Journal of Public Health, 99, 2034-2040. https://doi.org/10.2105/AJPH.2008.143099

Brauer, J. (n.d.). U.S. firearms sales: March 2020 unit sales show anticipated covid-19-related boom. Retrieved from 31 Jul 2020 http:// smallarmsanalytics.com/v1/pr/2020-04-01.pdf

Bresler, J., \& Beletsky, L. (2020). COVID-19, Incarceration, and the Criminal Legal System. 2020). Assessing Legal Responses to COVID-19. Boston: Public Health Law Watch.

Coibion, O., Gorodnichenko, Y., \& Weber, M. (2020). Labor markets during the covid-19 crisis: A preliminary view (No. 0898-2937). National Bureau of Economic Research.

Conner, A., Azrael, D., \& Miller, M. (2018). Public opinion about the relationship between firearm availability and suicide: Results from a national survey. Annals of Internal Medicine, 168, 153-155.

Cunningham, R. M., Walton, M. A., \& Carter, P. M. (2018). The major causes of death in children and adolescents in the United States. New England Journal of Medicine, 379, 2468-2475.

Czeisler, M. É., Lane, R. I., Petrosky, E., Wiley, J. F., Christensen, A., Njai, R., Weaver, M. D., Robbins, R., Facer-Childs, E. R., \& Barger, L. K. (2020). Mental health, substance use, and suicidal ideation during the COVID-19 pandemic-United States, June 24-30, 2020. Morbidity and Mortality Weekly Report, 69, 1049.

Dahlberg, L. L., Ikeda, R. M., \& Kresnow, M. (2004). Guns in the home and risk of a violent death in the home: Findings from a national study. American Journal of Epidemiology, 160, 929-936.

FACTS National Survey User Guide. (2020). Firearm Safety Among Children and Teens.

Galea, S., Merchant, R. M., \& Lurie, N. (2020). The mental health consequences of COVID-19 and physical distancing: The need for prevention and early intervention. JAMA Internal Medicine, 180, 817-818.

Gray, D. M., Anyane-Yeboa, A., Balzora, S., Issaka, R. B., \& May, F. P. (2020). COVID-19 and the other pandemic: Populations made vulnerable by systemic inequity. Nature Reviews Gastroenterology \& Hepatology, 17, 520-522.

Grossman, D. C., Mueller, B. A., Riedy, C., Dowd, M. D., Villaveces, A., Prodzinski, J., Nakagawara, J., Howard, J., Thiersch, N., \& Harruff, R. (2005). Gun storage practices and risk of youth suicide and unintentional firearm injuries. JAMA, 293, 707-714.

Hamilton, D., Lemeshow, S., Saleska, J. L., Brewer, B., \& Strobino, K. (2018). Who owns guns and how do they keep them? The influence of household characteristics on firearms ownership and storage practices in the United States. Preventive Medicine, 116, 134-142.

Hawes, M.T., Szenczy, A.K., Klein, D.N., Hajcak, G., \& Nelson, B.D. (2021). Increases in depression and anxiety symptoms in adolescents and young adults during the COVID-19 pandemic. Psychological Medicine, 1-9. https://doi.org/10.1017/S003329172 0005358

Hill, R.M., Rufino, K., Kurian, S., Saxena, J., Saxena, K., \& Williams, L. (2021). Suicide Ideation and Attempts in a Pediatric Emergency Department Before and During COVID-19. Pediatrics, 147(3), e2020029280. https://doi.org/10.1542/peds.2020-029280

Holmes, E. A., Oconnor, R. C., Perry, V. H., Tracey, I., Wessely, S., Arseneault, L., Ballard, C., Christensen, H., Silver, R. C., \& Everall, I. (2020). Multidisciplinary research priorities for the COVID19 pandemic: A Call for action for mental health science. The Lancet Psychiatry, 76, 547-560.

Kochanek, K., Murphy, S., Xu, J., \& Arias, E. (2019). National vital statistics report: Deaths, final data for 2017 (Volume 68, Number 9). Centers for Disease Control and Prevention.

Lang, B. J., \& Lang, M. (2020). Firearm Sales and the COVID-19 Pandemic. Available at SSRN 3593956.

McCarthy, J. (2014). More than six in 10 Americans say guns make homes safer. Gallup. November, 7.

Miller, M., Azrael, D., \& Hemenway, D. (2002). Firearm availability and unintentional firearm deaths, suicide, and homicide among 5-14 year olds. Journal of Trauma and Acute Care Surgery, 52, 267-275.

Miller, M., Azrael, D., Hemenway, D., \& Vriniotis, M. (2005). Firearm storage practices and rates of unintentional firearm deaths in the United States. Accident Analysis \& Prevention, 37, 661-667.

Monuteaux, M. C., Azrael, D., \& Miller, M. (2019). Association of increased safe household firearm storage with firearm suicide and unintentional death among US youths. JAMA Pediatrics, $173,657-662$.

Pirtle, W. N. L. (2020). Racial capitalism: A fundamental cause of novel coronavirus (COVID-19) pandemic inequities in the United States. Health Education \& Behavior.

Richters, J., \& Saltzman, W. (1990). Survey of exposure to community violence: Self report version. Rockville.

Schleimer, J. P., McCort, C. D., Pear, V. A., Shev, A., Tomsich, E., AsifSattar, R., Buggs, S., Laqueur, H. S., \& Wintemute, G. J. (2020). Firearm purchasing and firearm violence in the first months of the coronavirus pandemic in the United States. MedRxiv.

Vacha, E. F., \& McLaughlin, T. F. (2000). The impact of poverty, fear of crime, and crime victimization on keeping firearms for protection and unsafe gun-storage practices: a review and analysis with policy recommendations. Urban Education, 35, 496-510. https:// doi.org/10.1177/0042085900354006

van Dorn, A., Cooney, R. E., \& Sabin, M. L. (2020). COVID-19 exacerbating inequalities in the US. Lancet (London, England), 395, 1243.

Weinberger, D. M., Chen, J., Cohen, T., Crawford, F. W., Mostashari, F., Olson, D., Pitzer, V. E., Reich, N. G., Russi, M., \& Simonsen, L. (2020). Estimation of excess deaths associated with the COVID-19 pandemic in the United States, March to May 2020. JAMA Internal Medicine, 180, 1336-1344.

Wiktor, S. Z., Gallaher, M. M., Baron, R. C., Watson, M. E., \& Sewell, C. M. (1994). Firearms in New Mexico. Western Journal of Medicine, 161, 137.

Publisher's Note Springer Nature remains neutral with regard to jurisdictional claims in published maps and institutional affiliations. 